\title{
Cambio tecnológico y diferencial salarial en las regiones de México: un análisis de datos de panel para el sector servicios ${ }^{1}$ Mario Camberos C.*, Luis Huesca Reynoso** y David Castro Lugo ${ }^{\star \star *}$
}

\section{Resumen}

En este trabajo se realiza una aplicación de la hipótesis del cambio tecnológico sesgado para el sector servicio en las regiones de México. Se utilizan los microdatos de los censos económicos 1998, 2003 y 2008. Para probar la hipótesis del cambio tecnológico sesgado, se elaboran índices de diferenciales tecnológico y salarial, como también se acude al análisis de panel para mostrar la consistencia estadística de los resultados. Encontramos que la diferencia salarial mayor en el 2008, se da entre la capital y la región sur, que si bien tendió a reducirse a lo largo del periodo, que era cercana a 5 , al final, en el 2008, se redujo a 2.67 entre ambas regiones, en correspondencia con la reducción de la brecha tecnológica, tal vez debido al impacto de la crisis económica.

Palabras clave: cambio tecnológico sesgado, diferencial salarial, brecha tecnológica, regiones de México, análisis de panel.

\section{Abstract}

In this paper Hypothesis Biased Technological Change is applied for Mexican workers services sector, belonging several Mexican regions. Economics Census microdata, 1998, 2003 and 2008 are used. Hypothesis is proved with technological gaps, under consideration of different index and result statistics consistency by taking account panel analysis. Mayor wages differences at 2008 year were find out between Capital region and South one, about five hundred percent on 1998 year; but it was lower on 2008, two hundred percent. This result is in correspondence with diminishing technological gap, perhaps caused by economic crisis impact.

Keywords: biased technological change, salaries differences, technological gap, mexican economic regions, panel analysis.

1 Los autores agradecen el apoyo otorgado por el Conacyt para la realización de esta investigación como parte del Proyecto de Ciencia Básica (SEP 2008-01-106684).

* Investigador titular del Centro de Investigación en Alimentación y Desarrollo, A.C.- Hermosillo. Correo electrónico: mcamberos@ciad.mx

** Investigador titular del Centro de Investigación en Alimentación y Desarrollo, A.C.- Hermosillo. Correo electrónico: lhuesca@ciad.mx

***Profesor-investigador del Centro de Investigaciones Socioeconómicas de la Universidad Autónoma de Coahuila. Correo electrónico: david.castro@uadec.edu.mx 


\section{Introducción: El cambio tecnológico sesgado}

En la literatura más reciente sobre el mercado laboral se encontró en diferentes estudios para Estados Unidos que a raíz de una aceleración en la oferta de calificaciones, el rendimiento de los universitarios se redujo bruscamente durante la década de 1970; sin embargo, se incrementó durante los años de la década de 1980 en general para la mayoría de los países de la Organización para la Cooperación y el Desarrollo Económicos (OCDE), por efecto del aumento de la demanda de trabajo calificado sobre el no calificado, lo que dio lugar a la hipótesis del cambio tecnológico sesgado. Estos cambios han motivado a la literatura empírica a estudiar nuevamente la desigualdad, como dan cuenta diferentes estudios realizados sobre el tema para diferentes países.

\section{Aplicaciones de la hipótesis para Estados Unidos}

En esta línea de estudio Bound y Johnson (1992), Katz y Murphy (1992) y Acemoglu (1998 y 2002) demuestran que las diferencias salariales se deben al cambio tecnológico que privilegia la demanda del trabajo calificado, llamado así al trabajo que tiene preparación universitaria, lo cual le permite recibir una prima salarial sobre el no calificado. Otro conjunto de autores prueba también la hipótesis en un marco diferenciado considerando los mercados laborales de Estados Unidos y la Unión Europea en un marco institucional (Horstein, Krusel y Violante, 2004) y encuentran que la desigualdad salarial cambia muy poco en la Unión Europea, por las rigideces que impone el mercado laboral, a diferencia de lo ocurrido en Estados Unidos, donde los sindicatos han influido poco (Mosher, 2007).

Posteriormente, diferentes estudios han probado que la calificación multiplicada con el uso de equipo de cómputo permite sustituir tareas rutinarias por funciones no rutinarias (Autor et al., 1998; Acemoglu y Autor, 2010), resultados que han acotado la hipótesis, en el sentido de que es la demanda de trabajo que utiliza equipo de cómputo, por encima de la demanda de trabajadores que no lo utilizan, el factor que explica el incremento en la desigualdad salarial, como lo probaron Autor, Levy y Murnane (2003) para Estados Unidos; si bien Card y Di Nardo (2002) 
encontraron que en la década de 1990 la desigualdad salarial en dicho país, medida con la desviación estándar de los log, se mantuvo constante, a pesar del desarrollo de la tecnología de la informática; por lo tanto, éste es un factor más que compone el rompecabezas del problema llamado desigualdad.

\section{Evidencias para Chile, Colombia y México}

La hipótesis ha sido puesta a prueba también en países de América Latina, como lo hizo Gallego (2006) en Chile, quien encuentra aumentos en la demanda relativa de trabajadores calificados junto con la desigualdad salarial en las dos últimas décadas del siglo xx. Por su parte, Arango, Posada y Uribe (2005), para el caso de Colombia, descubrieron que en las dos últimas décadas del siglo xx se incrementaron más rápido los salarios de los trabajadores con mayor nivel educativo, como indicador de la calificación, respecto de los menos educados y por ello el diferencial salarial y el incremento en la desigualdad. En esta misma línea de investigación Torres (2002) observa en la industria española durante el periodo 1980-1992, utilizando distintas variables relacionadas con el cambio tecnológico, como la intensidad de uso del capital, el gasto en I+D y el uso de ordenadores, que existe correlación positiva entre la prima salarial de trabajo cualificado, considerado éste como el que desarrolla actividades administrativas.

Respecto del estudio del diferencial salarial para México, Cortez (2005), en una investigación para México y sus regiones en el periodo 1984-2000, con base en las Encuestas Nacionales de Ingreso y Gasto, observó que la dispersión salarial creció de 0.99 a 1.12 durante el periodo 1984 a 1998, pero de 1998 a 2000 cayó a 1.0. Igualmente, Meza (2005), utilizando la ENEu para los años 1988-1999, muestra la desigualdad salarial, medida con el log natural de la desviación estándar total, como también la diferencial salarial 75-25 del log del salario promedio de los percentiles 75 y 25; aunque disminuyó en la parte baja de la distribución medida con los percentiles 50-10.

Por su parte, Rodríguez, Huesca y Camberos (2011), con base en las Encuestas de Ocupación y Empleo (INEGI, 2008) de la última década del siglo xx y de la primera del siglo xxi y la clasificación de las actividades sectoriales para las regiones de México comprobaron que entre la región 
frontera, que posee mayor trabajo calificado y utiliza tecnología más moderna que la sur, en la que predominan actividades que hacen uso de tecnología tradicional, los salarios en promedio resultaron ser el doble, lo que abona a la añeja desigualdad regional en México. Finalmente, Abraham, para la India (2010), y Camberos y Huesca para México (2012), probaron la hipótesis con el uso de equipo de cómputo como indicador y encontraron también evidencias de aumentos en la desigualdad salarial.

Así, el trabajo tiene como objetivo mostrar la manera en que el cambio tecnológico, liderado por el uso del equipo de cómputo, afecta los diferenciales salariales en el sector servicios de las regiones de México en el periodo 1998-2008: ¿'homogeneizó los salarios entre las regiones?, o, por el contrario, ¿aumentaron las brechas salariales entre las misma?

El plan de exposición es el siguiente. En un primer apartado mostramos los aspectos teóricos en los que se sustenta este trabajo. Luego, analizamos la información estadística utilizada para demostrar empíricamente la relación entre el cambio tecnológico y el diferencial salarial. Después, elaboramos algunos índices para comparar las brechas tecnológica y salarial entre las regiones y sus cambios en el tiempo, para comprobar empíricamente si las brechas tecnológicas se corresponden con las salariales. En seguida aplicamos un modelo econométrico de panel para probar la confiabilidad estadística de la relación entre diferencial salarial y uso de equipo de cómputo como la variable explicativa, para demostrar que la relación no resulta espuria. Por último se muestran las conclusiones.

\section{El premio a la calificación y la habilidad}

Acemoglu (2002), un autor muy influyente en este tema, presenta un modelo que vincula los salarios a la oferta y la demanda generados por la frontera de posibilidades tecnológicas, considerando dos tipos de trabajadores, los calificados $H$ y los no calificados $L$ que son sustitutos perfectos y de eso dependen sus salarios. Supone también que el mercado laboral es competitivo.

El sesgo puede ilustrarse por medio de la figura 1, con la demanda relativa de trabajadores calificados contra la oferta de los mismos, H/L, en el equilibrio inicial en el punto A y un salario como $w$. Un aumento de 
la oferta de $H / L$ a $H / L$ ' mueve el punto de equilibrio de A a $\mathrm{B}$, bajando la demanda relativa de trabajadores calificados, lo que explicaría la caída del salario a $w$ '.

Pero esta tendencia de la caída de la demanda es contrarrestada por los cambios en la tecnología, que provocan un incremento relativo en la demanda de trabajo calificado en sustitución de trabajo no calificado, lo que impulsa el desplazamiento de la curva de la demanda de trabajo hacia arriba y por ello el incremento en el premio salarial por calificaciones de nuevo de $w^{\prime}$ a $w$, tal y como se muestra en la misma figura 1. El cambio tecnológico sesgado elevaría el salario hasta w' si la oferta no cambiara; pero ésta se ajusta rápidamente a la alza atraída por el aumento salarial mismo, llevando a la situación de equilibrio en el mercado de trabajo, a una tasa salarial igual o mayor a $w$, pero a un nuevo nivel de oferta $\mathrm{H} / \mathrm{L}$ en el punto C.

Figura 1. Demanda relativa por calificaciones

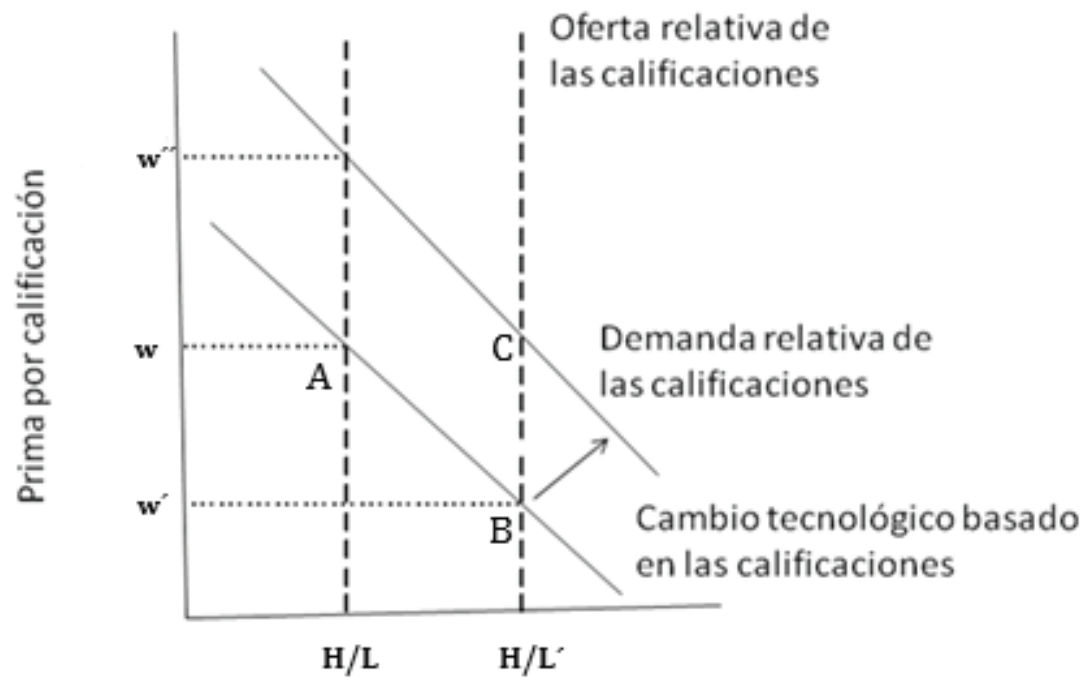

Fuente: Acemoglu, 2002.

Es éste el marco que nos permite plantear la siguiente hipótesis: el equipo de cómputo utilizado es el factor que explica el diferencial salarial en los servicios a favor de las regiones de México que registran el mayor índice de calificación en el periodo 1998-2008. 
Es importante destacar que el cambio tecnológico alcanza su verdadero impacto cuando llega a los sectores diferentes al que se genera, máxime cuando se trata del sector más grande de la economía, el que absorbe el mayor número de empleados y el que genera el mayor valor agregado, nos referimos a los servicios, pues ello implica, de suyo, que ha permeado a toda la sociedad.

Tomamos como indicador del cambio tecnológico, el valor del equipo de cómputo utilizado, junto con la inversión bruta en otro tipo de equipo. La inclusión de este segundo indicador del capital utilizado obedece a que éste también está asociado al cambio tecnológico, como parte del componente $(\mathrm{I}+\mathrm{D})$, con lo cual probaremos la consistencia del uso del equipo de cómputo como el factor que mayormente explica el diferencia salarial en México y sus regiones. La base de datos que utilizaremos es la derivada de los censos económicos 1998, 2003 y 2008. A lo largo del trabajo se hacen comparaciones con hallazgos encontrados por otras investigaciones.

\section{Las estadísticas de los servicios de las regiones de México}

Los censos económicos son una de las fuentes de información más confiable y aceptada para estudiar la economía mexicana y sus cambios en el tiempo. Los datos utilizados en este trabajo fueron elaborados con microdatos que proporcionaron las empresas para los censos de los años 1998, 2003 y 2008 (INEGI), procesados mediante el programa Stata 11. Para hacerlos comparables se tomó como base el año 2000 y se convirtió a valores per cápita cada una de las nueve ramas y 22 subramas del sector servicios de las 32 entidades integradas en seis regiones de acuerdo con la regionalización de Hanson (2004).

\section{Ajustes de los datos censales}

Los datos utilizados en las investigaciones deben ser de alta calidad, para garantizarlo, hacemos una limpieza de los mismos para comprobar la congruencia de las clasificaciones por uso de equipo de cómputo, formación bruta de capital y salarios per cápita, las tres variables más importantes que 
utilizaremos. Primero estimamos los valores totales para cada uno de los conceptos arriba citados, que fueron obtenidos como la suma de los microdatos proporcionada por cada empresa en cada una de las 22 subramas, que utilizamos para cada entidad, región y país. Después, los actualizamos a precios constantes del 2000 para hacerlos comparables. Encontramos que no existe la clasificación de un valor de equipo asignado a cada tipo de empleado como operarios, personal administrativo, propietarios y familiares, sino que se divide el valor del total del equipo en el año entre cada tipo de ellos, lo que da resultados sesgados per cápita, toda vez que el mismo valor del equipo varía considerablemente por tipo de personal ocupado para cada uno de los tres años.

Para salvar esta falla, dividimos el valor total del equipo entre el total de personal y así obtuvimos el indicador del valor del equipo de cómputo utilizado per cápita por cada subrama en cada uno de los años y las seis regiones de México. Finalmente, para hacerlos del todo comparables, elaboramos el otro indicador importante, el salario per cápita, que obtuvimos dividiendo la masa salarial entre el total de trabajadores de cada subrama.

\section{Las regiones}

Una manera de presentar los resultados que permite el análisis diferenciado y más amplio es mediante la división del país en regiones, puesto que facilita el combinar los cambios espaciales con los ocurridos en el tiempo y da lugar al empleo de técnicas de análisis más completas, como la de panel, que emplearemos en este trabajo. Para ello, consideramos la regionalización de Hanson (2004), basada en la contigüidad espacial de las entidades y acotada por las características económicas comunes predominantes en el espacio de las actividades que desarrollan los agentes económicos y sociales. El autor distingue seis regiones compuestas por los estados correspondientes y conformadas de la siguiente manera:

1. Frontera: Baja California, Chihuahua, Coahuila, Nuevo León, Sonora y Tamaulipas.

2. Norte: Aguascalientes, Baja California Sur, Durango, Nayarit, San Luis Potosí, Sinaloa y Zacatecas. 
3. Centro: Colima, Guanajuato, Hidalgo, Jalisco, Michoacán, Morelos, Puebla, Querétaro, Tlaxcala y Veracruz.

4. Capital: Distrito Federal y Estado de México.

5. Sur: Chiapas, Guerrero y Oaxaca.

6. Península: Campeche, Tabasco, Quintana Roo y Yucatán

\section{Brechas de calificación tecnológica y salarial}

En esta sección realizaremos una comparación entre las regiones a partir de índices elaborados con la región capital como punto de comparación, por ser ésta la que presenta los valores per cápita más elevados. La diferencia entre los índices nos permitirá medir las brechas de calificación de la mano de obra, tecnológica y salarial que existe entre las regiones y en el tiempo. Esta forma de análisis nos permitirá probar empíricamente la hipótesis del cambio tecnológico sesgado y su impacto en las diferencias salariales: un grado más alto de calificación de la mano de obra de la región capital, se corresponderá con una brecha tecnológica mayor por la intensidad del uso de equipo de cómputo, lo que deberá traducirse en un diferencial salarial más grande entre las regiones.

\section{La calificación de la mano de obra}

Un aspecto importante que debe quedar claro es que el uso de equipo de cómputo y el grado de calificación de la mano de obra están relacionados. Para demostrarlo construimos un índice de calificación para las regiones de México. Tomamos como proxy el porcentaje de matrícula de educación superior, respecto de la matrícula total, que equivale al indicador de estudios de colegio o universitarios que utilizaron Acemoglu y Autor (2010) para probar su hipótesis arriba mencionada. Con los resultados elaboramos el cuadro 1 que contiene en la primera columna el ranking de las regiones en donde la capital ocupa el primer lugar por tener el grado promedio más alto de escolaridad; por lo tanto de calificación. Después construimos un índice que normalizamos otorgándole a la capital el valor de 1; a continuación se dividen los grados de la capital entre los de cada región, la diferencia será 
el porcentaje de calificación mayor respecto de cada región. Los aspectos más relevantes son que la capital muestra un grado poco mayor que el de la frontera; pero resulta $50 \%$ más alto que el de la región centro y más del doble que el grado de calificación en el sur. Con estos hallazgos en mente, probaremos en los siguientes apartados si se corresponde el mayor grado de calificación, con mayor inversión por trabajador en equipo de cómputo y mayor diferencial salarial entre las regiones.

Cuadro 1. Ranking (2008) e índice de calificación por regiones de México

\begin{tabular}{|l|c|c|c|}
\hline \multicolumn{2}{|c|}{} & \multirow{2}{*}{$\begin{array}{c}\text { Índice } \\
\text { normalizado* }\end{array}$} & $\begin{array}{c}\text { Brecha** } \\
\text { de calificación }\end{array}$ \\
\cline { 1 - 2 } Ranking & Índice & & \\
1. Capital & 0.0997 & 100 & 1 \\
2. Frontera & 0.0954 & 95 & 1.05 \\
3. Norte & 0.0765 & 76 & 1.3 \\
4. Centro & 0.0688 & 69 & 1.5 \\
5. Sur & 0.0442 & 44 & 2.3 \\
6. Península & 0.0818 & 82 & 1.2 \\
\hline
\end{tabular}

*Se redondea a 100 para hacerlo más fácilmente comparable.

** Es el porcentaje de calificación mayor de la Capital respecto a cada una de las regiones.

Fuente: Estimaciones propias con base a Anexo Estadístico de Educación, II Informe del Presidente F. Calderón, 2008.

\section{La inversión en equipo de cómputo}

En el cuadro 2 se muestra la inversión en equipo de cómputo para cada una de las regiones; se pueden constatar las marcadas diferencias entre la capital con 69300 pesos per cápita y la frontera con 33825 pesos respecto de las regiones restantes, particularmente la del sur con 4859 y la del norte con 4326 pesos per cápita. Un análisis intertemporal nos muestra aumentos sustanciales entre 1998 y 2003 para la frontera, que alcanzó un volumen de inversión de 54826 pesos, y una caída drástica para la capital (22 036 pesos) y el centro (7 621 pesos); esto es, menos de una tercera par- 
te de la contabilizada al inicio del periodo. El resto de las regiones aumentó la inversión en equipo, si bien todavía muy por debajo de la frontera e incluso de la capital, a pesar de su sorprendente caída al final del periodo.

En los años 2003-2008, fue la frontera la que sufrió una caída drástica a 19361 pesos; por el contrario, la capital se recuperó hasta lograr 36106 y el centro 17445 pesos per cápita.

Si comparamos el periodo completo de 10 años, encontramos que el valor total del equipo de cómputo se redujo sustancialmente en la frontera a menos de $60 \%$ del valor de 1998, en la capital a poco más de $50 \%$ y en el centro a poco menos de $70 \%$. En el resto de las regiones se mantuvo en bajos niveles de inversión durante todo el periodo.

\section{Los salarios}

En el mismo cuadro 2 es fácil observar que las diferencias en inversión en equipo de cómputo se traducen en grandes diferencias salariales. Así, la capital registró en 1998 el salario más alto de 190269 pesos anuales por persona ocupada, secundándola la frontera con 75372 pesos, o sea, la mitad. En contraste, el salario en la región sur fue el más bajo con 35739 pesos y la península con 45292 pesos, diferencias salariales que están en

\section{Cuadro 2. Cambio tecnológico y salarios en las regiones de México}

Inversión en equipo de cómputo

Salarios

\begin{tabular}{lrr|r|r|r|rrr|}
\hline Regiones & Año 1998 & Año 2003 & Año 2003 & Año 1998 & Año 2003 & Año 2003 \\
\hline \multirow{2}{*}{ Frontera } & 33.825 & 54.816 & 19.361 & 75.372 & 99.707 & 80.337 \\
Norte & 4.379 & 6.5 & 6.828 & 46.489 & 57.927 & 58.811 \\
Centro & 25.407 & 7.621 & 17.445 & 57.871 & 60.134 & 58.661 \\
Capital & 69.39 & 22.036 & 36.106 & 190.269 & 105.34 & 105.721 \\
Sur & 4.859 & 7.746 & 8.775 & 35.739 & 43.265 & 39.487 \\
Penísula & 5.411 & 8.231 & 7.489 & 45.292 & 51.353 & 47.313 \\
\hline
\end{tabular}

Fuente: Estimaciones propias con base a Censos Económicos 1998, 2003 y 2008. 
línea con las encontradas por Meza para 1999 considerando las ciudades de la frontera (Tijuana, Ciudad Juárez y Monterrey) de 8 y 9 pesos por hora respecto de la región sur (Veracruz, Mérida y Orizaba), entre 4 y 5 pesos (2005).

En los siguientes años, los diferenciales de salarios siguieron prácticamente la tendencia de los cambios en el equipo de cómputo, observándose que al final del periodo todas las regiones resintieron los inicios de la crisis puesto que cayeron los salarios, principalmente en la frontera, o se mantuvieron casi constantes respecto de 2003, como en la capital, que ascendieron a 105721 - si bien en ésta cayeron fuerte respecto del inicio del periodo que alcanzó 190269 pesos por ocupado.

\section{Las brechas tecnológica y salarial}

Una dimensión del análisis que nos acerca más a la relación que nos interesa son los índices para medir las distancias entre el cambio tecnológico de las regiones y los salarios, verdaderas brechas que separan en el espacio y el tiempo las regiones de México. Para ello construimos el "índice de valor en equipo de cómputo por ocupado" (IEC), que obtenemos dividiendo el valor de la región capital $(\mathrm{C})$ entre cada una de las regiones: frontera $(\mathrm{F})$, norte $(\mathrm{N})$, centro $(\mathrm{Ce})$, sur $(\mathrm{S})$ y península $(\mathrm{P})$ en los tres años de registro, tomando a la capital como referencia por ser la de mayor inversión:

$$
\begin{gathered}
\mathrm{IEC}=\mathrm{Ct} / \mathrm{Rit} \\
\text { Con: } t \text { año, } R i \text { región }
\end{gathered}
$$

Del cuadro 2 se desprende que la capital observa un valor mayor en inversión en equipo de cómputo por ocupado que el resto de las regiones, toda vez que los valores del índice en todos los años y para todas las regiones son siempre superiores a 1, excepto la frontera en el año 2003. En este contexto, la región norte reflejaba la mayor brecha tecnológica para todo el periodo puesto que sus índices son los de mayor valor: 15.84, 3.39 y 5.29, seguida muy cerca por la región sur.

Al final del periodo, en 2008, todas las regiones habían reducido la brecha tecnológica respecto del inicio. Este hallazgo pudiera pensarse que 
es un síntoma de mejora en las regiones y de desigualdad salarial; sin embargo, no los es. Lo ocurrido es explicado más por la fuerte caída de la inversión en equipo de cómputo de la capital, que por el incremento de la misma en las regiones restantes, excepto la frontera. Es una convergencia hacia abajo que no beneficia al país. ¿Qué sucedió con la brecha salarial?

\section{La brecha salarial y el desarrollo regional (des)equilibrado}

Los resultados esperados de cuerdo con la hipótesis de trabajo es que a mayor brecha tecnológica entre la capital y las regiones, lo serán los diferenciales salariales entre las mismas, de tal suerte que el impacto del cambio tecnológico podrá contribuir al desarrollo equilibrado si reduce las diferencias entre las regiones. Para entenderlo mejor elaboramos el índice de diferencial salarial (IDS) tomando la región capital como referencia por ser la que observa las mayores percepciones per cápita, que fueron estimadas mediante la suma de las obtenidas por los operarios, administrativos, propietarios y familiares de cada región, dividida por el total del personal ocupado. Luego, se divide el salario per cápita de la capital (C), por ser la de mayor nivel, entre cada una de las regiones: frontera $(\mathrm{F})$, norte $(\mathrm{N})$, centro $(\mathrm{Ce})$, sur $(\mathrm{S})$ y península $(\mathrm{P})$ en los tres años de registro, tomando la región capital como referencia:

$$
\begin{gathered}
\text { IDS }=\text { Cst } / \text { Rsit } \\
\text { Con: } t \text { año, } R i \text {, región. }
\end{gathered}
$$

Los resultados se muestran en el cuadro 3. Se puede observar que las mayores brechas tecnológicas para el año de 1998 se corresponden con los mayores diferenciales salariales en los casos de las regiones norte, sur y península. En cambio, en las regiones frontera y centro es a la inversa: es mayor la brecha salarial que la tecnológica. En el 2003 esto se cumple para todas las regiones excepto para la frontera. Por último, en el 2008 en todas las regiones se cumple la parte de la comprobación empírica de la hipótesis que a una mayor brecha tecnológica mayor será el diferencial salarial. En lo que se refiere a los diferenciales salariales entre regiones, la capital presentaba en 1998 una brecha salarial 2.5 veces más respecto de 
Cuadro 3. Brechas tecnológica y salarial de las regiones de México

\begin{tabular}{|c|c|c|c|}
\hline \multicolumn{4}{c}{ Años } \\
\hline Regiones & 1998 & 2003 & 2008 \\
Frontera & & & \\
Tecnológica & 2.051 & 0.402 & 1.865 \\
Salarial & 2.524 & 1.056 & 1.316 \\
Norte & & & \\
Tecnológica & 15.845 & 3.39 & 5.288 \\
Salarial & 4.093 & 1.818 & 1.798 \\
Centro & & & \\
Tecnológica & 2.731 & 2.891 & 2.07 \\
Salarial & 3.288 & 1.752 & 1.802 \\
Capital & & & \\
Tecnológica & 1.000 & 1.000 & 1.000 \\
Salarial & 1.000 & 1.000 & 1.000 \\
Sur & & & \\
Tecnológica & 14.282 & 2.845 & 4.114 \\
Salarial & 5.324 & 2.435 & 2.677 \\
Península & & & \\
Tecnológica & 12.825 & 2.677 & 4.821 \\
Salarial & 4.201 & 2.051 & 2.235 \\
\hline
\end{tabular}

Fuente: Estimaciones propias con base a Censos Económicos 1998, 2003 y 2008.

la frontera, 4 veces más que el norte, 3.28 que el centro, 5.32 que el sur y 4.2 veces que la península; esto es, las mayores diferencias salariales en los servicios en 1998 fueron respecto del sur del país. Al final del periodo las diferencias tienden a reducirse de tal forma que para la frontera fueron de 1.31 veces, norte 1.79 , centro 1.80 , sur 2.67 y península 2.23; sin embargo, las mayores diferencias siguieron siendo entre la capital y el sur.

Estos resultados referidos a los diferenciales salariales entre las regiones son similares a los hallazgos de Cortez (2005) basados en los datos de 
las ENIGH y estimados con base en una ecuación minceriana, MCO (con heteroscedasticidad), en los que se muestra que un trabajador con educación universitaria en el estado de Guerrero gana 28.5\% menos que el promedio nacional; mientras que un trabajador de Baja California con ese mismo nivel gana $29.5 \%$ más.

Además, el mismo autor encuentra que Baja California y el Distrito Federal son los estados que presentan salarios superiores a la media en todas las categorías educativas; Nuevo León y Chihuahua presentan los mayores diferenciales salariales con educación universitaria, que correspondería al trabajo calificado, y los trabajadores de San Luis Potosí, Hidalgo, Guerrero, Oaxaca, Chiapas, Zacatecas, Puebla, Tlaxcala, Veracruz y Yucatán reciben salarios por debajo de la media. De éstos, Guerrero, Hidalgo y Chiapas presentan los salarios relativos más bajos (Cortez, 2005).

Estos datos nos llevan a concluir que el impacto del cambio tecnológico ha contribuido a un desarrollo regional desequilibrado (Carrillo, 2001). ¿Qué se puede concluir de este análisis? Que empíricamente se cumple la hipótesis que relaciona positivamente el cambio tecnológico y el diferencial salarial para la mayoría de las regiones y los años. ¿Es esta conclusión correcta? No necesariamente. El problema que plantea la verificación empírica de las hipótesis, es que éstas pueden ser fácilmente falsificadas con un caso que las contradiga. Por ello es necesario acudir a las pruebas estadísticas basadas en modelos reconocidos, como haremos en seguida.

\section{El análisis con datos de panel}

El tratamiento con datos de panel es el adecuado para las investigaciones en las que se dispone de pocas observaciones en el periodo de tiempo analizado, pero suficientes en cada uno de los años. En nuestro caso, el periodo abarca 10 años: 1998-2008, con observaciones para sólo tres años: 1998, 2003 y 2008, pero 132, para cada uno de ellos; por lo tanto, permite combinar el análisis longitudinal con el de corte transversal (Yaffee, 2003; Dougherty, 2007; Frees, 2004; Johnston, 1984) que garantiza resultados más robustos.

El punto central del trabajo es demostrar que el valor de la inversión en equipo de cómputo (ln ec), que representa una proxy del cambio tec- 
nológico, utilizado en 22 ramas, explica los niveles salariales (ln w) y las diferencias en las seis regiones; así como también demostrar la consistencia de la variable explicativa, para lo cual incluimos la formación bruta de capital (ln fbc) y finalmente las regiones y los años como variables dummies. Los resultados serán analizados a la luz de un conjunto de pruebas estadísticas que se sugieren para el caso por autores reconocidos como Greene (1999), Johnston (1984), Gujarati (1998), Dougherty (2007) y Yaffee (2003); mientras que para la aplicación del programa estadístico Stata nos basamos en Baum (2006). El procedimiento a seguir es el siguiente:

1. Se corre una regresión mco con todos los datos de panel que incluye los años $t$, las regiones $i$, las subramas $r$ y las variables $j$.

2. Se corrige la heteroscedasticidad con MCG.

3. Se aplica la prueba del multiplicador de Lagrange (ML) BreuschPagan para detectar el problema de la existencia de correlación de la variable explicativa y datos no observados Cov (Xit; Uit) $\neq 0$ (Montero, 2007), por medio de la cual se determinará si es procedente el análisis de datos de panel.

4. Se incluye la prueba de Hausman para elegir entre el método de efectos fijos o aleatorios.

5. Se corre el modelo completo con dummies de cada una de las regiones y los años para probar su influencia en los resultados y la consistencia de la variable explicativa.

La ecuación elemental para estimar la regresión con datos agrupados es la siguiente:

$$
y_{\mathrm{it}}=\alpha+\beta_{\mathrm{j}} X_{\mathrm{it}}+u_{\mathrm{it}}
$$

en nuestro caso, transformamos los datos con ln para ajustar mejor los resultados y obtener elasticidades:

$$
\ln \mathrm{w}_{\mathrm{it}}=\alpha+\beta_{1} \operatorname{lnec}_{1 \mathrm{it}}+\beta_{2} \ln f b c_{2 \mathrm{it}}+u_{\mathrm{it}}
$$

donde:

lnw: logaritmo natural del salario.

$\alpha$ : valor constante que no varía con las regiones ni los años. 
lnec: logaritmo natural del valor del equipo de cómputo.

lnfbc: logaritmo natural de formación bruta de capital.

$u$ : variables no observadas, que hipotéticamente no deberían estar correlacionadas con las variables explicativas.

\section{Estimaciones con mínimos cuadrados ordinarios (MCO)}

Los resultados para todos los métodos de ajuste que realizamos se sintetizan en el cuadro 4. Se observa en la segunda columna que los MCO son estadísticamente consistentes. Los valores de los coeficientes de las variables independientes son diferentes a cero y el valor per cápita del equipo de cómputo (ln_per_eq) 0.1703 , la variable explicativa relevante de la hipótesis, es mucho más alto que el de formación bruta de capital 0.1043. Los errores estándar son relativamente pequeños de tal suerte que los valores que arrojan las pruebas $t$ para los parámetros individuales son altamente significativos. La prueba $\mathrm{F}(2,392)=66.04$ Prob $>\mathrm{F}=0.0000$ rechaza la hipótesis nula de homoscedasticidad; el modelo registra heteroscedasticidad, resultado que era de esperarse por la diversidad de regiones.

\section{Estimaciones con mínimos cuadrados generalizados (MCG)}

El siguiente paso consiste en corregir el problema de heteroscedasticidad, para ello se corre la regresión MCG heteroscedástico, con las seis regiones como grupos, cuyos resultados aparecen en el cuadro 4 en la columna 3. Los coeficientes son diferentes de cero y el ln del equipo de cómputo aumenta su valor a 0.1815; en tanto que el parámetro de la fbc disminuye ligeramente y la constante mantiene prácticamente su valor. La prueba de Wald, de igualdad de parámetros, refuta la hipótesis, es decir, hay diferencias significativas.

\section{La prueba del multiplicador de Lagrange (ML) Breusch-Pagan}

Esta prueba, del ML, se aplica para determinar si existe un componente inobservable asociado a la varianza de cada individuo, Cov (Xit, $U$ it) $=0$ 
Cuadro 4. Relación salario, valor del equipo de cómputo y formación bruta de capital ajustada con varios métodos

\begin{tabular}{|c|c|c|c|c|c|c|}
\hline In salario & $M C O(1)$ & $M C G(2)$ & $M L-B P(3)$ & $E F(4)$ & $E A(5)$ & $\begin{array}{c}\text { Dummies } \\
\text { Año y Reg (6) }\end{array}$ \\
\hline ln eq. com | & 0.1703342 & 0.1815786 & 0.2060132 & 0.184466 & 0.1703342 & 0.2348823 \\
\hline$(\mathrm{t}, \mathrm{z})$ & 8.75 & 9.12 & 9.67 & 8.99 & 8.75 & \\
\hline ln formbru | & 0.1043697 & 0.0977494 & 0.1161989 & 0.0475189 & 0.1043697 & 0.0489969 \\
\hline$(\mathrm{t}, \mathrm{z})$ & 4.54 & 4.27 & 5.07 & 1.96 & 4.54 & \\
\hline constante & 3.28978 & 3.291816 & 3.192732 & 3.35383 & 3.28978 & 3.544597 \\
\hline$(t, z)$ & 58.27 & 58.99 & 56.15 & 60.71 & 58.27 & \\
\hline Núm. Obs. & 395 & 395 & 395 & 395 & 395 & 395 \\
\hline $\mathrm{R}^{2}$ & 0.252 & & & 0.2408 & 0.252 & 0.3956 \\
\hline Dentro & & & & 0.2285 & 0.2127 & 0.3028 \\
\hline Entre & & & & 0.3496 & 0.6499 & 1 \\
\hline $\mathrm{F}(2,392)$ & 66.04 & & 85 & 9.11 & & \\
\hline chi2 & & 139.49 & 137 & & 132.09 & 251.6 \\
\hline rho $\left(\mathbf{u}_{-} \mathbf{i}\right) \neq 0$ & & & 0.140974 & 0.137104 & 0 (supuesto) & 0 (supuesto) \\
\hline $\operatorname{corr}\left(\mathrm{u} \_\mathrm{i}, \mathrm{Xb}\right)$ & & & & 0.0695 & 0 (supuesto) & 0 (supuesto) \\
\hline
\end{tabular}

Fuente: Estimaciones propias con base a los Censos Económicos 1998, 2003, 2008 y Programa Stata, 2009.

(Montero, 2007) (subrama y región en nuestro trabajo), que sesgara los resultados MCO (Montero, 2007:2). O como ocurre para muchos conjuntos de datos en los que se espera encontrar correlación positiva cuando se examina $\left[\mathrm{y}_{\mathrm{i} 1}, \mathrm{y}_{\mathrm{i} 2}, \mathrm{y}_{\mathrm{iTi}}\right]$ (Frees, 2004). La prueba se realiza utilizando el estadístico $X^{2}$ (chi2): cuando el valor calculado es mayor que el estadístico de tablas, se rechaza la hipótesis nula de ausencia de correlación; por lo tanto, procede la aplicación del método de análisis de datos de panel (Dougherty, 2007). 
En la columna 4 se muestran los resultados de la prueba. Los valores de los parámetros son estadísticamente significativos diferentes de cero, con errores estándar bajos y valores $t$ altos; además las pruebas chi2(1) = 137.57, rho $=0.14097456,\left(u_{-} i\right) \neq 0$ y Prob $>F=0.0000$, significan que los errores están correlacionados y hay efecto de las variables no observadas en las variables independiente del modelo; por lo tanto, se rechaza la hipótesis nula. Resultado que confirma el hallazgo de la prueba de Wald, arriba descrita; por lo tanto es preferible el análisis de panel.

\section{El análisis de datos de panel: efectos fijos y efectos aleatorios}

La selección del método de análisis de efectos a seguir requiere la aplicación del modelo que contempla la ecuación para efectos fijos (Dougherty, 2007; Montero, 2007):

$$
y_{\mathrm{it}}=\alpha_{\mathrm{i}}+\beta_{\mathrm{j}} X_{\mathrm{it}}+u_{\mathrm{it}}
$$

donde $\alpha_{i}$ es conocido como el efecto no observado, porque recoge el impacto conjunto de las variables no observadas sobre $y_{\mathrm{i}}$ en la regresión (Dougherty, 2007) y es igual a $\left(\alpha+v_{\mathrm{i}}\right)$; de donde se desprende que el error $\left(\varepsilon_{\mathrm{i}}=v_{\mathrm{i}}+u_{\mathrm{it}}\right)$ se descompone en una parte fija, constante para cada región, y otra aleatoria que cumple con los requisitos MCO (Montero, 2007).

Los resultados aparecen en la columna 5 para efectos fijos, entre grupos. El coeficiente de la variable de la hipótesis $=0.18446$ y de signo positivo confirma la relación establecida, con una alta significación estadística. Los cambios en la media de las variables independientes explican más de los cambios del salario (between), que los individuales (within). Los valores de las pruebas corr $\left(u_{-}\right.$i, Xb) $=0.0695$ y la de todos $\operatorname{los} u_{-} \mathrm{i}=0: \mathrm{F}(5,387)$ $=9.11$ y Prob $>\mathrm{F}=0.0000$, no rechazan hipótesis nula de la correlación de los cambios en ui entán con los regresores; por lo tanto, los estimadores son consistentes (Baum, 2006). Veamos ahora si el análisis de efectos aleatorios es consistente y además eficiente. 


\section{Efectos aleatorios}

Cuando las variables de interés son constantes para cada individuo, efectos fijos no son recomendables porque cada variable no puede ser incluida (Dougherty, 2007). Si bien para efectos aleatorios se aplica la misma ecuación de efectos fijos (2) (Montero, 2007), con $\alpha_{\mathrm{i}}=\left(\alpha+v_{\mathrm{i}}\right)$, ahora $v_{\mathrm{i}}$, en lugar de ser un valor fijo para cada individuo y constante a lo largo del tiempo, es una variable aleatoria con una varianza $\operatorname{Var}\left(v_{\mathrm{i}}\right) \neq 0$. Este enfoque supone que es posible tratar cada una de las variables no observadas como derivada aleatoriamente de una distribución dada. Si éste es el caso, $\alpha_{i}$ puede ser tratada como variable aleatoria, de donde deriva su nombre.

En la columna 6 se presentan los hallazgos y se observa el valor del coeficiente de la variable de la hipótesis $=0.17033$ y el signo positivo, con una alta significación estadística, que confirma la relación establecida por nuestra hipótesis. También destaca que el valor $\mathrm{R}^{2}$ entre $=0.6499$, explica más de los cambios salariales que $\mathrm{R}^{2}$ dentro $=0.2197$.

Igualmente de acuerdo a los resultados de la prueba de Wald chi2 $(2)=132.09$ Prob $>$ chi2 $=0.0000$, se rechaza la hipótesis nula de independencia de los errores corr $\left(u_{-} i, X\right)=0$ (supuesta).

¿Cuál de los dos tipos de análisis es el más adecuado?

\section{La prueba de Hausman para seleccionar entre efectos fijos $y$ efectos aleatorios}

La prueba parte de la hipótesis nula: las diferencias entre los coeficientes estimados por ambos métodos no son sistemáticos; es decir, no existe correlación entre el error y los regresores. Si el valor de la prueba es alto y el p-valor bajo < 0.05 (Gujarati, 1998), se rechaza la hipótesis y es preferible aplicar efectos fijos (Montero, 2007).

Los hallazgos se muestran el cuadro 5, en el que se comparan los coeficientes de ambos y de acuerdo al estimador chi2 $(2)=0.00$, Prob > chi2 $=1.0000$ se concluye que las diferencias encontradas no son sistemáticas; esto es, no existe correlación estadísticamente significativa entre los errores y los regresores, detectada con la prueba del multiplicador de 
Cuadro 5. Resultados de la prueba de Hausman

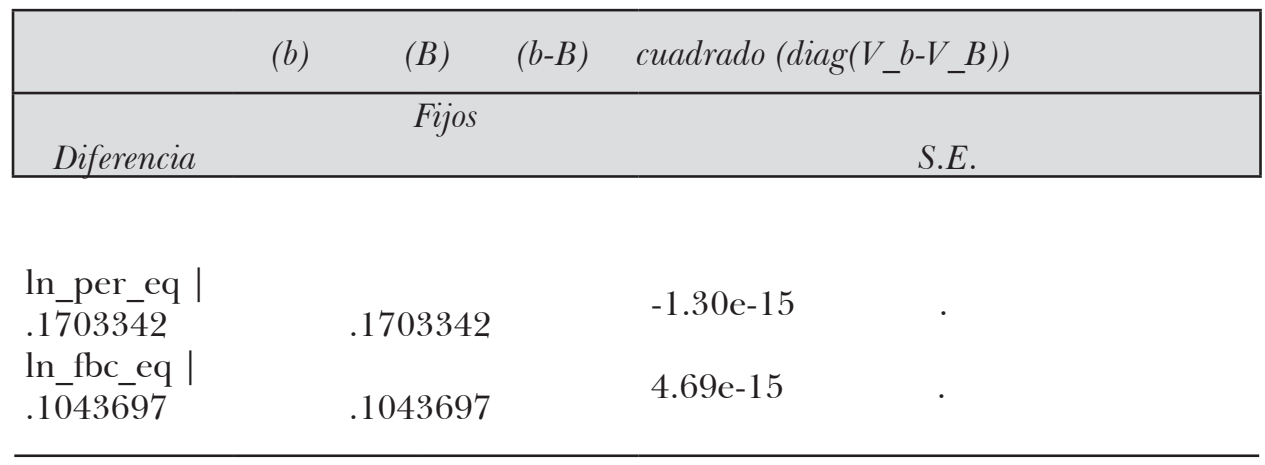

Prueba Ho: Dif. en coef. no son sistemáticas, chi2(2) $=0.00$,Prob $>$ chi2 $=1.0000$

Fuente: Estimaciones propias con base a los Censos Económicos 1998,2003, 2008 y Stata, 2009.

Lagrange. Por lo tanto, los resultados con efectos aleatorios son consistentes y eficientes (Bauman, 2006) y se elige el análisis de panel de efectos aleatorios.

\section{El efecto de las regiones y el tiempo:} la consistencia de la variable de la hipótesis

Para terminar probamos el efecto de las regiones y el tiempo con el modelo de efectos aleatorios. Para ello incluimos las variables dummies. Los resultados que se muestran en la última columna del cuadro 4 y en el cuadro 6 indican que la capacidad explicativa del equipo de cómputo aumenta a 0.2348 respecto a $\mathrm{MCO}=0.1703, \mathrm{MCG}=0.1815 \mathrm{y} \mathrm{EF}=0.1844$, así como con los mismos $\mathrm{EA}=0.1703$ sin las dummies. Las regiones $(\mathrm{r} 1, \mathrm{r} 2, \mathrm{r} 3, \mathrm{r} 4$, r5, r6) y el tiempo (a1, a2, a3) son estadísticamente significativas puesto que sus errores estándar son pequeños y tienen efecto en la explicación. Los signos negativos de las dummies para las regiones hacen saber que en promedio las regiones observaron salario menor que el de la frontera en promedio para prácticamente toda la primera década del siglo xxı. El 


\section{Cuadro 6. Relación salario y equipo de cómputo ajustada con efectos aleatorios, regiones y años}

\begin{tabular}{|c|c|c|c|c|c|c|}
\hline$l n \_w \_e q$ & Coef. & Err. Est. & $z$ & $P>|z|$ & \multicolumn{2}{|c|}{ [95\% inter conf] } \\
\hline ln_per_eq & 0.2348823 & 0.0232324 & 10.11 & 0.000 & .1893476 & .280417 \\
\hline ln_fbc_eq & 0.0489969 & 0.0245678 & 1.99 & 0.046 & .0008449 & .0971489 \\
\hline r2 & -0.2713979 & 0.0950063 & -2.86 & 0.004 & -.4576067 & -.085189 \\
\hline r3 & -0.1956353 & 0.0942989 & -2.07 & 0.038 & -.3804579 & -.0108128 \\
\hline $\mathrm{r} 4$ & -0.2509242 & 0.1013267 & -2.48 & 0.013 & -.4495209 & -.0523276 \\
\hline r5 & -0.6265603 & 0.100435 & -6.24 & 0.000 & -.8234091 & -.4297114 \\
\hline r6 & -0.5169452 & 0.1007327 & -5.13 & 0.000 & -.7143776 & -.3195129 \\
\hline $\mathrm{a} 2$ & 0.2426878 & 0.0695211 & 3.49 & 0.000 & .1064289 & 3789468 \\
\hline a3 & -0.2211503 & 0.0784839 & -2.82 & 0.005 & -.3749758 & -.0673247 \\
\hline _cons & 3.544597 & 0.0974609 & 36.37 & 0.000 & 3.353577 & 3.735617 \\
\hline sigma_u & \multicolumn{6}{|l|}{0} \\
\hline sigma_e & \multicolumn{6}{|l|}{.53876843} \\
\hline rho & \multicolumn{6}{|c|}{$0 \quad$ (fraction of variance due to $u_{-} \_$i) } \\
\hline
\end{tabular}

Núm. de obs. $=396 ; \operatorname{corr}\left(u_{-} \mathrm{i}, \mathrm{X}\right)=0$ (assumed); Wald chi2(9) $=251.6$ Prob $>$ chi2 $=0.0000$ $\mathrm{R}^{2}$ : within $=0.3028$, between $=1.0000$, overall $=0.3953$

Regiones: r1 Frontera, r2 Norte, r3 Centro, r4 Capital, r5 Sur y r6 Península.

Fuente: Estimaciones propias con base a los Cen. Econ. 1998, 2003 y 2008 y Programa Stata 2009. 
signo positivo del año 2 representa el aumento salarial que se registró en el 2003 respecto de 1998, y el signo negativo del año 3 representa la caída del salario del año 2008. El valor de la varianza $\left(u_{-} i=0\right.$, rho $\left.=0\right)$ significa que se elimina la correlación entre las variables no observadas y las explicativas, lo que pudiera deberse a que se elimina la heterogeneidad no observada (Dougherty, 2007), resultado que se consigue con la inclusión como dummies a las regiones y los años.

\section{Conclusiones}

La búsqueda de explicaciones acerca del funcionamiento de los mercados ha llevado en las últimas décadas a enfocarse en el impacto que produce el avance tecnológico en la demanda y el diferencial salarial, a la luz de la hipótesis del cambio tecnológico sesgado, cuyo versión más actual resulta en el incremento de la demanda de trabajo calificado que utiliza equipo de cómputo sobre el tipo de trabajo que no hace uso del mismo como explicación del diferencial salarial.

En este trabajo hemos analizado una aplicación de la hipótesis y se encontró que las diferencias en la utilización del equipo de cómputo o brecha tecnológica resultaron mayores en las regiones que presentan los más altos niveles de calificación como la capital y la frontera norte, observándose la diferencia más grande entre la capital y la región sur, que si bien era cercana a 5 al inicio del periodo, se redujo en el 2008 a 2.67. La diferencias salariales se correspondieron con las brechas tecnológicas representadas por el valor del equipo de cómputo utilizado por persona empleada en cada región.

Luego se demuestra que la relación es estadísticamente confiable, probando la hipótesis con un método econométrico de datos de panel, toda vez que Mínimos Cuadrados Ordinarios presentó problemas de heteroscedasticidad que se corrigió con MCG, pero persistía el problema estadístico de alta correlación entre las variables no observadas y las explicativas.

Para solventar el problema se aplicó la prueba del multiplicador de Lagrange y se encontró que existía un componente inobservable asociado a la varianza de cada individuo, Cov (Xit, $U$ it) $=0$, por lo que procedía aplicar el análisis de panel de datos. En seguida, mediante la prueba de 
Hausman se determinó utilizar efectos aleatorios y se pudo comprobar que cuando se incluyen dummies para las regiones y los años, el parámetro de la variable de la hipótesis, el equipo de cómputo, arroja mayor valor y consistencia: pasó de $\mathrm{MCO}=0.1703$ a PANEL $=0.2349$.

Por último, es necesario enfatizar que los resultados mostrados en este trabajo sobre las diferencia entre las regiones no hacen más que corroborar las desigualdades encontradas en otros estudios que utilizan otro tipo de indicadores económicos y de desarrollo social: el rezago del sur y la península con respecto de la capital y la frontera, que da cuenta también del desarrollo desequilibrado entre las regiones de México.

\section{Bibliografía}

Abraham, V., (2010), The Effect of Information Technology on Wage Inequality: Evidence from India Manufacturing Sector, documento de trabajo 437, Centre's Website $(<$ www.cds.edu $>$ ).

Acemoglu, D. y D. Autor (2010), Skills, Tasks and Technologies: Implicatiion for Employment and Earnings, documento de trabajo 16082, NBER, Cambridge, MA.

Acemoglu, D. (2002), "Technical Change, Inequality, and the Labor Market", Journal of Economic Literature, vol. 40, núm. 1, pp. 7-72.

_ P. Aghion y G. L. Violante (2001), "Desunionization, Technical Change and Inequality", Carnegie-Rochester Conference Series on Public Policy, núm. 55, pp. 229-264.

— (1998), Changes in Unemployment and Wage Inequality: An Alternative Theory and some Evidence, documento de trabajo 6658, National Bureau of Economic Research, pp. 1-39.

Arango, L. E., C. E. Posada y J. D. Uribe (2005), "Cambios en la estructura de los salarios urbanos en Colombia, 1984-2000”, Lecturas de Economía, núm. 63, julio-diciembre, Universidad de Antioquia, Colombia, pp. 7-39.

Autor, D. H., F. Levy y R. J. Murnane (2003), "The Skill Content of Recent Technological Change: An Empirical Exploration", Quarterly Journal of Economics, vol. 116, núm. 4.

Autor, D. H., L. F. Katz y A. B. Krueger (1998), "Computing Inequality: Have Computers Changed the Labor Market?”, Quartely Journal of Economics, vol. CXIII, núm. 4, pp. 1170-1213.

Baum, Cristopher F. (2006), An Introduction to Modern Econometrics Using Stata, Stata Press, Texas, Estados Unidos. 
Berman, Eli, J. Bound y G. Zvi (1994), "Changes in the Demand for Skilled Labor within U.S. Manufacturing Industries”, Quarterly Journal of Economics, núm. 109, pp. 367-398.

Bound, J. y G. Johnson (1992), "Changes in the Structure of Wages in the 1980s: An Evaluation of Alternative Explanations", American Economic Review, núm. 83, pp. 371-392.

Calderón, F. (2008), II Informe de Gobierno, Anexo Estadístico de Educación, México.

Camberos M. y L. Huesca (2012), "Technical Change and Labor Market in Mexico: Anticipating the Shappe Occupation and Qualification for a Better Assessment", Abstracts, The International Conference on Innovation Methods for Innovation Management and Policy (Im2012), Beijing, China, 21-24 de mayo, pp. 85-86.

Card, D. y J. E. DiNardo (2002), "Skill-Biased Technological Change and Rising Wage Inequality: Some Problems and Puzzles", Journal of Labor Economics, vol. 20, núm. 4, pp. 733-784.

Carrillo H. M. (2001), "La teoría clásica de la convergencia y la realidad del desarrollo regional en México", Problemas del Desarrollo, vol. 32, núm. 127, UNAM-IIE, México, pp. 107-134

Cortez W. W. Y. (2005), "Dispersión y estabilidad de las diferencias salariales interestatales en México, 1984-2000”, Investigación Económica, vol. LXIv, núm. 253, julio-septiembre, pp. 123-158.

Dougherty, C. (2007), Introduction to Econometrics, Harvard University Press, Cambridge.

Frees, E. F. (2004), Longitudinal and Panel Data, Cambridge University Press, Reino Unido.

Gallego, Francisco (2006), Skill Premiun in Chile: Studying the Skill Bias Techical Change Hypothesis in the South, documento de trabajo 363, pp. 1-46.

Goldin, Claudia y L. Katz (2010), The Race between Education and Technology, Cambridge, Reino Unido.

Greene, W.H. (1999), Análisis Econométrico, Prentice Hall Iberia, Madrid.

Gujarati, D. (1998), Econometría, Prentice Hall, Madrid.

Hanson, Gordon (2004), "What has Happened to Wages in México since NAFTA? Implications for Hemispheric Free Trade", en Dany Rodrick, Alan Taylor y Andrés Velasco (eds.), FTAA and Beyond: Prospects for Integration in the Americas, Cambridge Harvard University Press, Estados Unidos.

Horstein, Andreas, Per Krusel y Giovanni L. Violante (2004), The Effects of Technical Change on Labor Market Inequalities, documento de trabajo, 04-08, FRB of Richmond, pp. 1-129, CEPR DP 5025.

INEGI (2008), Encuesta Nacional de Ocupación y Empleo, México.

—— (1998, 2003 y 2008), Censos Económicos, México. 
Johnston, John (1984), Econometric Analysis, McGraw Hill, Nueva York.

Katz, L. y K. Murphy (1992), "Changes in Relative Wages: Supply and Demand Factors", Quarterly Journal of Economics, vol. CVIII, pp. 35-78.

Meza, Liliana (2005), "Mercados laborales locales y desigualdad salarial en México”, El Trimestre Económico, vol. Lxxiı, núm. 285, enero-marzo, pp. 133178.

Montero, G. R. (2007), Efectos fijos o variables: test de especificación, Granada, España. Mosher, J. S. (2007), "U.S. Wage Inequality, Technological Change and Decline Union Power", Politics E Society, núm. 35, pp. 225-263.

Murphy, K. y F. Welch (1989), "Wage Premiums for College Graduates: Recent Growth andPossible Explanations", Educational Researcher, AERA, 18 de mayo, pp. 17-26.

Rodríguez, Reyna, L. Huesca y M. Camberos (2011), "Mercado laboral, inequidad salarial y cambio tecnológico”, Frontera Norte, núm. 45, El Colegio de la Frontera Norte, Tijuana, México, enero-junio, pp. 7-33.

Stata Program (2009), Version 11, Stata Press, College Station, Texas, Estados Unidos.

Torres, X. V. (2002), “Dispersión salarial y cambio tecnológico en la industria española”, Investigaciones Económicas, vol. xxvi, núm. 3, pp. 551-571.

Yaffe, R. (2003), A Primer for Panel Data Analysis, New York University, Nueva York.

Artículo recibido el 2 de noviembre de 2011. Segunda versión aprobada el 10 de enero de 2012. 